\title{
Rendimento acadêmico no Ensino Superior: variáveis pessoais e socioculturais do estudante
}

\section{Academic performance in Higher Education: personal and socio- cultural variables of the student}

\author{
${ }^{1}$ Bruna Casiraghi bruna@casiraghi.com.br \\ ${ }^{2}$ Leandro da Silva Almeida \\ ${ }^{3}$ Evely Boruchovitch \\ ${ }^{4}$ Júlio Cesar Soares Aragão
}

\section{RESUMO}

O rendimento acadêmico (RA) se configura como a forma de mensurar o aprendizado ao longo da formação e é determinado por fatores relacionados ao discente, às instituições de ensino e aos docentes, sendo comumente utilizado como critério de avaliação do sucesso acadêmico. A presente investigação objetiva avaliar os fatores relacionados ao RA de estudantes do Ensino Superior e se configura como estudo censitário, realizado em uma Instituição privada de Ensino Superior brasileira. Diversas informações foram coletadas, por meio de formulário eletrônico, junto dos estudantes: opção de curso e instituição, cursos, idade, sexo, período cursado, grau de instrução de pai e mãe, renda familiar mensal e autopercepção do RA. Dados relativos ao RA foram obtidos no banco de dados institucional, considerando o Coeficiente de Rendimento, estimado pela média de todas as notas finais obtidas pelo aluno em todas disciplinas cursadas. Participaram 264 estudantes de 19 cursos, com média de idades de 22,25 (DP=5,57) anos, sendo 166 (62.9\%) do sexo feminino. A variação de RA foi significativa em relação às áreas de conhecimento, sexo, faixa etária e autopercepção do rendimento. Não foi significativa quanto ao grau de instrução de pai e mãe, quando segmentados em todos os níveis, entretanto, a escolaridade da mãe torna-se relevante quando estratificada a partir dos oito primeiros anos de escolaridade. Os resultados permitem inferir que os alunos possuem uma correta percepção de seu RA e que a construção deste rendimento é multifatorial. Considerações e implicações para a prática são tecidas com base nos resultados.

Palavras-chave: Rendimento acadêmico. Ensino Superior. Autopercepção.

\begin{abstract}
Academic achievement (AA) is a means of measuring learning over graduation and is determined by factors related to students, educational institutions and teachers, being a commonly used criterion in academic success assessment. The present investigation intents to evaluate the factors related to the AR of higher education students and is configured as a census study, carried out in a private Brazilian Higher Education Institution. Using an electronic form, variety of information was collected from students: course and institution option, courses, age, sex, period of enrolment, level of education of father and mother, monthly family income and self-perception of the AA. Data related to the AA were obtained from the institutional database, considering the Overall Score, estimated by the average of all final grades obtained by the student in all subjects taken. From 19 courses, 264 students participated, with an average age of $22.25(S D=5.57)$ years, and $166(62.9 \%)$ females. The variation in AA was significantly related to areas of knowledge, gender, age range and score self-perception. There was no relation to father and mother level of education, when all levels were considered, however, mother's education became relevant when stratified from eight years of schooling. These results allow us to infer that students have a correct perception of their AR and that this performance construction is multifactorial. Considerations and implications for practice are made based on the results.
\end{abstract}

Keywords: Academic achievement. Higher education. Self perception.

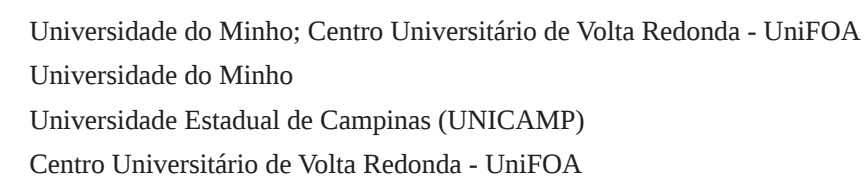




\section{INTRODUÇÃO}

O Ensino Superior no Brasil experimentou ampla expansão entre o final do século XX e início do século XXI. Esse movimento ocorreu tanto na esfera pública quanto privada, embora marcadamente na segunda e com sérios questionamentos quanto à qualidade dos serviços ofertados (SAVIANI, 2011). Esta expansão, com efeito, decorreu em grande medida por causa do aumento do número de instituições de Ensino Superior e da multiplicação dos cursos ofertados, o que nem sempre corresponde a uma devida avaliação dos recursos e condições adequadas de funcionamento.

Em um país com uma desigualdade histórica e mobilidade social restrita, a oportunidade de frequentar um curso de nível superior pode ser vista como um importante mecanismo de criação de oportunidades e melhoria da qualidade de vida da população. Além disso, em um mundo cada vez mais dependente de mão de obra qualificada e do desenvolvimento e aprimoramento científico, o impacto da educação superior na sociedade como um todo é inegavelmente positivo (MARTINS; MARTINS, 2019).

A despeito da notória ampliação da oferta e da inserção de mecanismos que garantam o acesso da população ao Ensino Superior, tais esforços devem ser vistos como uma conquista ainda incipiente, dado que o ingresso no Ensino Superior não garante a formação de todos indivíduos admitidos no âmbito universitário (ALMEIDA et al., 2012; BARBOSA, 2019; BORGES; RIBEIRO, 2019). O percurso acadêmico de um estudante inclui dificuldades que se estendem desde o âmbito sócio econômico até questões individuais, perpassando a aprendizagem e o rendimento acadêmico (RA). As taxas de insucesso e de evasão são elevadas, e também não afetam da mesma forma os diversos grupos socioculturais. Minorias étnicas e grupos socialmente mais desfavorecidos apresentam taxas mais reduzidas de conclusão das suas formações académicas.

No Brasil, o Censo do Ensino Superior (INSTITUTO NACIONAL DE ESTUDOS E PESQUISAS EDUCACIONAIS ANÍSIO TEIXEIRA, 2019) aponta que, no ano de 2018, foram registradas 8.450 .755 matrículas ativas, 1.290.017 matrículas trancadas e 2.187.411 matrículas desvinculadas (sem vínculo por motivo de abandono, evasão, desligamento ou transferência) o que indica que quase 30\% do total de matrículas não se encontravam ativas no momento da realização do censo. Dentre as matrículas ativas, 3.445.935 são ingressantes e 1.264.288 concluintes, números que, por si só, não representam abandono ou insucesso, contudo apontam para uma disparidade entre o volume de alunos que ingressam nas universidades e o número de formados no mesmo período.

Os trabalhos científicos adotam, na maioria das vezes, como critérios de avaliação do sucesso acadêmico o desempenho alcançado, usualmente expresso por meio das notas, e a conclusão do curso (ARAÚJO, 2017; ISAZA VALENCIA, 2014; RICHARDSON; ABRAHAM; BOND, 2012; VALADAS; ARAÚJO; ALMEIDA, 2014). Desempenho e conclusão são fatores intimamente associados, mas não completamente interdependentes, tendo em vista que o rendimento (pelo menos o mínimo) exigido é condição inequívoca para a conclusão do curso, mas o baixo rendimento não é o fator único e determinante para a não conclusão ou para a evasão. Kuh, Kinzie e Buckley (2006) destacam que a conclusão do curso é a medida mais definitiva para aferir o sucesso acadêmico e que as notas ao longo do curso permitem acompanhar o processo, juntamente com dados qualitativos sobre o empenho e o sentimento de cumprimento e realização.

O RA se configura como a forma mais frequente de mensurar o aprendizado ao longo da formação e é determinado por fatores relacionados ao discente, às instituições de ensino e aos docentes (FERREIRA, 2009; SOUSA; FERREIRA; MIRANDA, 2019). No Ensino Superior, o RA, além de significar a promoção do aluno nas disciplinas ou períodos, representa a aquisição de conhecimentos, competências e habilidades profissionais (BECERRA-GONZÁLEZ; MARTÍNEZ, 2015). Segundo Bertolin e Marcon (2015), são raras as pesquisas sobre os fatores determinantes do desempenho dos estudantes no Ensino Superior no Brasil. Esses autores ainda apontam que o maior impacto no rendimento advém do contexto familiar, social, econômico e cultural dos estudantes. Os trabalhos sobre a temática revelam diferentes fatores associados ao bom RA no Ensino Superior (Bac- 
caro \& Shinyashiki, 2014; Cunha \& Carrilho, 2005; Elias et al., 2009), destacando aspectos relacionados aos estudantes, como a saúde física e mental, o grau de instrução da família ou questões relacionadas às estratégias de estudo, autorregulação ou motivação para a aprendizagem; aspectos relacionados aos professores, como as metodologias de ensino, relacionamento professor-aluno ou estratégias de avaliação, e os aspectos da instituição, como, por exemplo, apoio financeiro, estrutura disponível, apoio psicopedagógico.

A falta de consenso na literatura a respeito da relevância de cada fator no RA (BRANDT, 2017), longe de invalidar a consistência dos estudos, reforça o caráter multidimensional do RA perante diferentes fatores que detém relação direta com o desempenho, mas que também tecem, entre si, uma imbricada rede de interação cujo impacto poderá ser modulado, em maior ou menor grau, dependendo da sinergia existente. É importante destacar que o RA é resultado da busca de aferir a aprendizagem do aluno, contudo não é possível garantir que a nota represente as competências adquiridas. O processo avaliativo é complexo e depende de uma multiplicidade de influências que variam cronológica e geograficamente entre estudantes, docentes, disciplinas, cursos e instituições, de acordo com estratégias e materiais utilizados (RODRIGUES JÚNIOR, 2009).

Ao lidar com dados obtidos pelos estudantes (questionários ou entrevistas), uma das dificuldades frequentemente relatada por investigadores desta temática é a obtenção dos dados relativos ao RA. Consequentemente, muitas pesquisas consideram o rendimento percebido pelos estudantes e não o seu rendimento efetivo, dificuldade que conseguimos superar neste estudo pois os serviços académicos da instituição onde a pesquisa foi realizada forneceu as classificações escolares dos estudantes da amostra. Neste sentido, o presente estudo tem como objetivo avaliar os fatores relacionados ao RA de estudantes do Ensino Superior e relacionar a autopercepção do RA com o coeficiente de rendimento acadêmico, aferido pela média das notas dos alunos em todas as disciplinas cursadas.

\section{MÉTODO}

\section{Participantes}

Foram obtidos dados de 264 estudantes universitários, provenientes de uma instituição privada do interior do Rio de Janeiro - Brasil. As idades variaram de 18 a 62 anos, com média de 22,29 anos (DP = 5,54), de ambos os sexos, sendo a maior parte do sexo feminino (62,9\%). Alunos de todos os anos de formação foram incluídos, considerando que os cursos possuem diferentes tempos de integralização, sendo 101 (38,3\%) do primeiro ano, $52(19,7 \%)$ do segundo, $28(10,6 \%)$ do terceiro, $56(21,2 \%)$ do quarto, $24(9,1 \%)$ do quinto e $3(1,1 \%)$ do sexto (somente curso de Medicina possui seis anos), e das três áreas de conhecimento: 39,7\% da área de humanas, compreendida pelos cursos de Direito, Administração, Ciências Contábeis, Licenciatura em Ciências Biológicas, Jornalismo, Publicidade e Propagando e Serviço Social; 33\% da área da saúde, dos cursos de Enfermagem, Graduação em Ciências Biológicas, Educação Física, Medicina, Nutrição e Odontologia; e 27,3\% da área de exatas, alunos dos cursos de Design, Engenharia Ambiental, Engenharia Civil, Engenharia de Produção, Engenharia Elétrica e Engenharia Mecânica. Quanto à renda familiar em salários mínimos, que equivalem a R\$998,00 ou US\$ 273,00 em 2019 (ILO, 2020), 41 estudantes (15,5\%) apresentavam renda familiar de até dois salários mínimos, 92 (34,8\%) de dois a cinco salários mínimos, 35 (13,3\%) de cinco a oito salários mínimos, 23 (8,7\%) referiram renda superior a oito salários mínimos e 73 (27,7\%) estudantes não sabiam ou referiram não informar sua renda familiar.

\section{Instrumentos}

A coleta dos dados foi realizada por meio de formulário eletrônico e compilado em um banco de dados. Embora a abrangência do banco seja maior, o presente estudo analisou os seguintes dados: cursos, idade, sexo, período cursado, grau de instrução de pai e mãe, renda familiar mensal, se o curso era sua primeira opção, se a 
instituição era a primeira opção e autopercepção do RA. Dados relativos ao RA foram obtidos no banco de dados institucional, considerando o Coeficiente de Rendimento.

\section{Procedimentos}

A presente investigação foi realizada em 2019 com estudantes de diversos cursos de uma Instituição de Ensino Superior situado no interior do Estado do Rio de Janeiro. Os dados fazem parte de um projeto de pesquisa maior em curso. A pesquisa foi submetida ao SISNEP e autorizada sob o Registro 96352018.5.0000.5237. A variação do RA segundo diferentes fatores foi avaliada pela Análise de Variância (ANOVA), recorrendo ao software IBM/SPSS, versão 26.0.

\section{Resultados}

Ao serem questionados sobre a escola do seu curso, 138 estudantes (52,3\%) informam que não estão cursando sua primeira opção de carreira e $149(54,4 \%)$ referem que a instituição que frequentam era sua primeira opção. Este dado pode ser explicado, entre outros motivos, pela disponibilidade de cursos em horários noturnos ou meio período, que viabiliza a coexistência de uma vida acadêmica em conjugação com o exercício de uma atividade laboral. Indagados sobre a necessidade de sair da casa dos pais para ingressar no Ensino Superior, 217 $(82,2 \%)$ informaram que não houve necessidade de mudança, o que indica que a maioria desses alunos optaram por uma instituição de ensino que não exigisse mobilidade geográfica. Quanto ao grau de instrução dos pais (Tabela 1), a maior taxa reporta-se ao ensino médio completo, tanto do lado paterno (75; $28,4 \%$ ), quanto materno (80; 30,3\%), seguida do Ensino Superior completo, 65 (24,6\%) dos pais e 79 (29,9\%) das mães.

Tabela 1- Escolaridade dos pais

\begin{tabular}{lcccc}
\hline & \multicolumn{2}{c}{ Paterno } & \multicolumn{2}{c}{ Materno } \\
\hline Grau de Instrução & $\mathbf{N}$ & $\mathbf{\%}$ & $\mathbf{N}$ & $\mathbf{\%}$ \\
\hline Mestrado e Doutorado & 9 & 3,4 & 9 & 3,4 \\
\hline Ensino Superior completo & 65 & 24,6 & 79 & 29,9 \\
\hline Ensino Superior incompleto & 26 & 9,8 & 37 & 14 \\
\hline Ensino Médio completo & 75 & 28,4 & 80 & 30,3 \\
\hline Ensino Médio incompleto & 25 & 9,5 & 15 & 5,7 \\
\hline Ensino Fundamental completo & 31 & 11,7 & 17 & 6,4 \\
\hline Ensino Fundamental incompleto & 18 & 6,8 & 21 & 8 \\
\hline Sem escolaridade & 2 & 0,8 & 1 & 0,4 \\
\hline
\end{tabular}

Fonte: elaborado pelos autores.

Para fins das análises estatísticas, as idades dos estudantes foram distribuídas nos seguintes estratos: Menores de 20 anos (90; 34,1\%), entre 20 e 25 anos (134; 50,7\%) e maiores de 25 anos (40; 15,2\%). Em relação ao rendimento académico dos estudantes da amostra (RA), obteve-se uma média de 7,4 na escala de 1 a 10 , situando-se a mediana em 7,7 e a moda na classificação de 8,0. A comparação da média do RA com as diversas variáveis, permitindo considerar subgrupos de estudantes dentro da amostra, está apresentado na Tabela 2. 
Tabela 2- Comparação de médias do RA

\begin{tabular}{|c|c|c|c|c|}
\hline & Média & $\mathbf{N}$ & Desvio padrão & Sig \\
\hline SEXO & & & & $\mathrm{p}=0,028$ \\
\hline Feminino & 7,56 & 166 & 1,23 & \\
\hline Masculino & 7,18 & 98 & 1,59 & \\
\hline ÁREA DE CONHECIMENTO & & & & $\mathrm{p}=0,001$ \\
\hline Exatas & 7,12 & 72 & 2,04 & \\
\hline Humanas & 7,8 & 105 & 0,96 & \\
\hline Saúde & 7,21 & 87 & 1 & \\
\hline IDADE (POR ESTRATO) & & & & $\mathrm{p}=0,017$ \\
\hline$<20$ & 7,74 & 90 & 1,08 & \\
\hline $20-25$ & 7,31 & 134 & 1,47 & \\
\hline$>25$ & 7,08 & 40 & 1,59 & \\
\hline SE O CURSO ERA PRIMEIRA OPÇÃO & & & & $\mathrm{p}=0,704$ \\
\hline Não & 7,39 & 138 & 1,43 & \\
\hline Sim & 7,45 & 126 & 1,33 & \\
\hline SE A INSTITUIÇÃO ERA PRIMEIRA OPÇÃO & & & & $\mathrm{p}=0,132$ \\
\hline Não & 7,57 & 115 & 1,17 & \\
\hline Sim & 7,31 & 149 & 1,52 & \\
\hline SAIU DA CASA DOS PAIS/ MUDOU-SE & & & & $\mathrm{p}=0,695$ \\
\hline Não & 7,44 & 217 & 1,45 & \\
\hline Sim & 7,35 & 47 & 1,04 & \\
\hline RENDA MENSAL FAMILIAR & & & & $\mathrm{p}=0,208$ \\
\hline até 02 salários mínimos & 7,21 & 41 & 1,34 & \\
\hline de 02 até 05 salários mínimos & 7,6 & 92 & 1,06 & \\
\hline de 05 até 08 salários mínimos & 7,54 & 35 & 2,08 & \\
\hline Superior a 08 salários mínimos & 7,69 & 23 & 1,36 & \\
\hline Não sei/ Prefiro não informar & 7,17 & 73 & 1,34 & \\
\hline
\end{tabular}

Fonte: elaborado pelos autores.

A comparação da média do RA mostrou-se significativa quando consideramos a variável gênero dos estudantes $(F=-2,21, p<0,05)$, área de conhecimento $(F=7,09, p \leq 0,01)$ e idade $(F=4,15, p<0,05)$. Não se observaram diferenças estatisticamente significativas quando se consideram os estudantes frequentando ou não o curso de primeira opção e a instituição de primeira opção, ainda que registre um RA mais elevado por parte dos estudantes a frequentar um curso de $1^{\mathrm{a}}$ opção, invertendo-se essa situação em relação à frequência de instituição de primeira opção. O ter saído ou não de casa dos pais para frequentar o Ensino Superior não diferencia o RA dos estudantes, registando-se uma ligeira subida por parte dos estudantes que continuaram a viver com os pais. Relativamente à análise de RA segundo o rendimento familiar dos estudantes também não se verifica uma diferenciação das médias tomando os quatro extratos constituídos. Mesmo havendo variabilidade, ela não é linear considerando a progressão na renda familiar, mesmo quando estratificado em um menor número de faixas de renda (por exemplo, menor ou maior que dois salários mínimos). É possível que a frequência de uma instituição de Ensino Superior privada defina um certo padrão de rendimento econômico dos estudantes, seja apoiado na família, seja em alguma atividade profissional que acumulam com os estudos.

Centrando-nos nas variáveis em que se observa uma diferença estatisticamente significativa nas médias de RA, observa-se que a média do RA do sexo feminino $(7,56)$ é superior ao do masculino $(7,18)$. Em relação à idade, verifica-se uma diminuição da média de RA à medida que avançamos no grupo etário dos estudantes. Esta 
diminuição é linear em função da idade, assim como a média dos estudantes mais novos (abaixo de 20 anos) é mais alta, seguida do grupo entre 20 e 25 anos, sendo que o grupo de estudantes acima de 25 anos apresenta uma média mais baixa. Com relação a área de conhecimento, os estudantes da área de Humanas apresentam a média mais alta, seguidos dos da área da Saúde e de Exatas, o que terá seguramente a ver com características específicas do currículo e do processo avaliativo de cada curso.

Ao examinar o grau de instrução dos pais (Tabela 3), não houve relação significativa no grau de escolaridade dos pais com variações do RA. Todavia, salta aos olhos as diferenças existentes entre todas as categorias e os RA de universitários cujas mães não tem nenhuma escolaridade ou, ainda, que desconhecem a escolaridade das próprias mães.

Tabela 3- Comparação de médias por grau de escolaridade dos pais

\begin{tabular}{|c|c|c|c|c|c|c|c|}
\hline Grau de instrução do pai & Média & $\mathbf{N}$ & DP & Grau de instrução da mãe: & Média & $\mathbf{N}$ & DP \\
\hline sem escolaridade & 7,5 & 2 & 1,98 & sem escolaridade & 6,8 & 1 & \\
\hline ensino fundamental ( $1^{\circ}$ grau) incompleto & 7,08 & 18 & 1,76 & ensino fundamental ( $1^{\circ}$ grau) incompleto & 6,98 & 21 & 1,82 \\
\hline ensino fundamental $\left(1^{\circ}\right.$ grau $)$ completo & 7,54 & 31 & 1,3 & ensino fundamental ( $1^{\circ}$ grau $)$ completo & 7,38 & 17 & 1,05 \\
\hline ensino médio ( $2^{\circ}$ grau) incompleto & 7,29 & 25 & 0,94 & ensino médio ( $2^{\circ}$ grau) incompleto & 7,31 & 15 & 1,17 \\
\hline ensino médio ( $2^{\circ}$ grau) completo & 7,49 & 75 & 1,41 & ensino médio ( $2^{\circ}$ grau) completo & 7,59 & 80 & 1,17 \\
\hline superior incompleto & 7,19 & 26 & 0,97 & superior completo & 7,35 & 79 & 1,59 \\
\hline superior completo & 7,73 & 65 & 1,12 & superior incompleto & 7,66 & 37 & 1,17 \\
\hline mestrado ou doutorado & 6,87 & 9 & 2,59 & mestrado ou doutorado & 7,68 & 9 & 0,96 \\
\hline não sei informar & 6,75 & 13 & 2,05 & não sei informar & 6,1 & 5 & 2,2 \\
\hline
\end{tabular}

Sig. grau de instrução paterno $p=0,261$, materno $p=0,275$

Fonte: elaborado pelos autores.

Cabe ressaltar que a ampla gama de categorias utilizadas torna difícil atingir a especificidade almejada para o presente estudo. Por esse motivo, optamos por reagrupar as categorias agregando as escolaridades paterna e maternas em um número de categorias menor que 8 anos (escolaridade desconhecida, nenhuma escolaridade ou fundamental incompleto) e 8 anos ou mais (fundamental completo em diante) conforme estudos já realizados internacionalmente e em território brasileiro (ANAZAWA et al., 2016). Esta modelagem permitiu identificar efeito positivo na RA de alunos com mães que cursaram a partir de 8 anos de ensino fundamental (7,49 vs 6,8, $\mathrm{p}=0,015)$ não havendo o mesmo resultado para a escolaridade paterna (7,48 vs 6,98, $\mathrm{p}=0,05)$.

Os resultados do RA também variaram consideravelmente de acordo com a autopercepção do aluno (Tabela 4), demonstrando que os estudantes conseguem ter uma boa dimensão de seus resultados e categorizá-los de uma forma bem próxima da realidade, ou seja, alunos que se consideraram bem abaixo da média para passar (nesta instituição um resultado igual ou superior a 7,0) apresentaram RA de 4,87, com médias crescentes até a percepção de estar bem acima da média, que apresentou RA de 8,54. 
Tabela 4- Comparação de média do RA e autopercepção do RA

\begin{tabular}{lcccc}
\hline \multicolumn{1}{c}{ Suas notas, na maioria das vezes, ficam } & N & Min./Max. & Média & Erro Desvio \\
\hline bem abaixo da nota média que o seu curso exige para passar & 4 & $4,5-5,5$ & 4,88 & 0,48 \\
\hline abaixo da nota média que o seu curso exige para passar & 14 & $4,2-6,8$ & 5,74 & 0,77 \\
\hline em torno da nota média que o seu curso exige para passar & 112 & $4,0-9,3$ & 6,94 & 1,3 \\
\hline acima da nota média que o seu curso exige para passar & 105 & $5,6-9,4$ & 7,94 & 1,03 \\
\hline bem acima da nota média que o seu curso exige para passar & 29 & $7,5-9,5$ & 8,55 & 1,21 \\
\hline \multicolumn{1}{c}{ Total } & 264 & $4,0-9,5$ & 7,42 & 1,38 \\
\hline
\end{tabular}

$\mathrm{F}=29,34, \mathrm{p}<0,001$

Fonte: elaborado pelos autores.

\section{DISCUSSÃO}

O Ensino Superior cumpre hoje nas sociedades modernas e mais desenvolvidas um papel decisivo na promoção do rendimento econômico e qualidade de vida das pessoas. Ao mesmo tempo que o tecido econômico e social requerem mão de obra mais qualificada científica e tecnicamente, também as políticas de equidade e igualdade de oportunidades de formação dos diversos grupos socioeconômicos fizeram expandir o Ensino Superior. Neste momento, grupos sociais e étnicos bastante diferenciados, inclusive aqueles que durante décadas ficaram afastados do Ensino Superior, acedem a este segmento de escolarização, devendo ser preocupação das instituições não apenas garantir o acesso, mas assegurar o sucesso e a conclusão da formação da generalidade dos estudantes, sem que nessa taxa incida negativamente a origem social menos privilegiada (ALMEIDA et al., 2012; BARBOSA, 2019; BORGES; RIBEIRO, 2019) .

Nesta perspectiva, o presente estudo procurou avaliar o impacto de certas variáveis dos estudantes no seu rendimento académico (RA), em particular variáveis mais diretamente relacionadas com a sua origem socioeconômica e com o processo de transição para o Ensino Superior, em particular se acedeu ou não a um curso e a uma instituição de sua primeira opção. Os resultados obtidos mostram uma média mais elevada de RA entre os estudantes de sexo feminino, de faixa etária abaixo dos 20 anos e da área de Ciências Humanas, não apresentando relação com frequentarem curso ou instituição de primeira escolha, saída da casa dos pais e renda familiar. Também em vários outros estudos, em nível internacional, se observa melhor rendimento académico por parte das alunas, sugerindo estar mais concentradas nos seus estudos e recorrerem a métodos mais eficazes de aprendizagem (ALEMÁN; TRÍAS; CURIONE, 2011; ALMEIDA et al., 2006; BARAHONA, 2014; OYARZÚN ITURRA et al., 2012). Em relação a idade, estudantes mais novos podem ter os seus hábitos de estudo e seus conhecimentos académicos anteriores mais próximos face aos estudantes mais velhos, que poderão ter deixado de estudar há alguns anos. Além disso, tendencialmente os estudantes mais velhos exercem outras funções ou assumem mais responsabilidades familiares e sociais, podendo isso dificultar a frequência às aulas e a aprendizagem.

Relativamente à área científica do curso, são apontadas maiores dificuldades dos estudantes nas áreas das Ciências e da Tecnologias, passando essas dificuldades de rendimento académico pela natureza da estrutura curricular dos cursos e pelos métodos de avaliação, ou ainda a diferente predominância por sexo nos diferentes cursos e seus agrupamentos por áreas científicas (ALEMÁN; TRÍAS; CURIONE, 2011; BARAHONA, 2014; OYARZÚN ITURRA et al., 2012).

A não diferenciação do RA em função da escolha do curso e da instituição pode decorrer da instabilidade dessa mesma vinculação por parte dos estudantes. Algumas vezes, estudantes que entram no curso e instituição de sua primeira escolha se desiludem, enquanto que aqueles que não os elegeram como primeira opção acabam 
por encontrar colegas, infraestruturas, docentes e serviços que agradam, invertendo percepções iniciais de menor vinculação (OSTI; ALMEIDA, 2019). No Brasil, as diferenças de oferta e qualidade entre instituições públicas e privadas influenciam na eleição de um curso ou instituição como de primeira escolha, tendo em vista que as opções que os estudantes vislumbram dependem da renda familiar, da qualidade da educação básica, da oferta de curso na região em que reside ou o turno em que os cursos são oferecidos, permitindo ou não associar o estudo com trabalho. Em uma realidade em que o Ensino Superior público oferece vagas insuficientes e a concorrência para ingresso é alta, alguns estudantes almejam uma vaga, mas a maioria não a alcançam, enquanto outros nem consideram isso como uma possibilidade (PIOTTO; NOGUEIRA, 2016).

Embora os dados referentes a escolaridade paterna e materna, de forma geral, não apresentem diferenças significativas no RA, essa associação é relevante quando agregadas em função dos primeiros oito anos de escolaridade da mãe, reforçando o impacto da escolarização feminina na qualidade de vida e no desenvolvimento dos filhos. Esta associação, podendo ser mais significativa na infância, deixa antever o papel da mãe na estruturação das atividades escolares dos alunos ao longo da infância e da adolescência, podendo traduzir-se em hábitos diferenciados de estudo e de comprometimento com as tarefas de aprendizagem no momento de acesso ao Ensino Superior (ALVES et al., 2016; NORO et al., 2019).

A autopercepção do rendimento acadêmico apresentou correlação significativa com as médias dos estudantes pesquisados, sugerindo que, na ausência ou impossibilidade de aquisição do RA, esta variável possa ser tomada como um substituto com algum grau de associação e fiabilidade. Este dado é tanto mais importante quando se verificam dificuldades dos investigadores acederem ao rendimento acadêmico efetivo dos estudantes por parte dos serviços administrativos das instituições, inclusive alegando questões éticas para a não cedência dessa informação.

\section{CONSIDERAÇÕES FINAIS.}

O presente estudo teve como objetivo avaliar os fatores relacionados ao RA de estudantes do Ensino Superior e se configura como estudo censitário, realizado em uma Instituição privada de Ensino Superior brasileira. Os dados da presente investigação reforçam a multidimensionalidade de fatores associados ao RA e apontam para a necessidade de estudos que avaliem o comportamento desta variável e comparem os resultados apresentados por diferentes cenários acadêmicos. Esta diversidade de variáveis merece ser estudada e diferenciada nos seus níveis de impacto, tentando desta forma apoiar os diretores e responsáveis pedagógicos das instituições na implementação de medidas de apoio ao sucesso acadêmico e à conclusão dos cursos dos seus estudantes.

\section{REFERÊNCIAS}

ALEMÁN, M. J.; TRÍAS, D.; CURIONE, K. Orientaciones motivacionales, rendimiento académico y género en estudiantes de bachillerato. Ciências Psicológicas, v. 5, n. 2, p. 159-166, nov. 2011.

ALMEIDA, L. et al. Democratização do acesso e do sucesso no ensino superior: uma reflexão a partir das realidades de Portugal e do Brasil. v. 17, n. 3, p. 22, 2012.

ALMEIDA, L. S. et al. Acesso e sucesso no Ensino Superior em Portugal: questões de género, origem sóciocultural e percurso académico dos alunos. Psicologia: Reflexão e Crítica, v. 19, n. 3, p. 507-514, 2006.

ALVES, A. F. et al. Desempenho Cognitivo na Infância: A Mãe e o Meio Urbano fazem a Diferença. Psicologia: Teoria e Pesquisa, v. 32, n. 3, 2016. 
ANAZAWA, L. et al. A Loteria da Vida: Examinando a Relação entre a Educação da Mãe e a Escolaridade do Jovem com Dados Longitudinais do Brasil. Policy Paper, v. 22, p. 47, 2016.

ARAÚJO, A. M. Sucesso no Ensino Superior: Uma revisão e conceptualização. Revista de estudios e investigación en psicología y educación, v. 4, n. 2, p. 132-141, 2017.

BACCARO, T. A.; SHINYASHIKI, G. T. Relação entre desempenho no vestibular e rendimento acadêmico no ensino superior. Revista Brasileira de Orientação Profissional, v. 15, n. 2, p. 165-176, dez. 2014.

BARAHONA, P. Factores determinantes del rendimiento académico de los estudiantes de la Universidad de Atacama. Estudios pedagógicos (Valdivia), v. 40, n. 1, p. 25-39, 2014.

BARBOSA, M. L. O. Democratização ou massificação do Ensino Superior no Brasil? | Higher Education in Brazil: democratization or massification? Revista de Educação PUC-Campinas, v. 24, n. 2, p. 240-253, 26 jun. 2019.

BECERRA-GONZÁLEZ, C. E.; MARTÍNEZ, L. M. R. Motivación, autoeficacia, estilo atribucional y rendimiento escolar de estudiantes de bachillerato. REDIE. Revista Electrónica de Investigación Educativa, v. 17, n. 3, p. 79-93, 2015.

BERTOLIN, J. C. G.; MARCON, T. O (des)entendimento de qualidade na educação superior brasileira - Das quimeras do provão e do ENADE à realidade do capital cultural dos estudantes. Avaliação: Revista da Avaliação da Educação Superior (Campinas), v. 20, n. 1, p. 105-122, mar. 2015.

BORGES, G. F. S.; RIBEIRO, E. A. A expansão da educação superior brasileira a partir dos anos 90: democratização ou massificação? Revista Triângulo, v. 12, n. 1, p. 103-118, 18 abr. 2019.

BRANDT, J. Z. Ensino superior no Brasil: fatores influenciadores no desempenho acadêmico dos alunos de graduação. Tese de Mestrado—Braga: Universidade do Minho, 2017.

CUNHA, S. M.; CARRILHO, D. M. O processo de adaptação ao ensino superior e o rendimento acadêmico. Psicologia Escolar e Educacional, v. 9, n. 2, p. 215-224, dez. 2005.

ELIAS, A.; AZEVEDO, V.; MAIA, Â. Saúde e rendimento académico nos estudantes da Universidade do Minho: percepção de áreas problemáticas. 2009. Disponível em: <https://repositorium.sdum.uminho.pt/>. Acesso em: 10 jul. 2019

FERREIRA, M. Determinantes do Rendimento Académico no Ensino Superior. Revista Internacional d'Humanitats, v. 15, p. 55-60, abr. 2009.

FILIPA ALVES, A. et al. Cognitive performance and academic achievement: How do family and school converge? European Journal of Education and Psychology, v. 10, n. 2, p. 49 , 3 jan. 2018.

ILO. Statistics on WagesILOSTAT, 2020. Disponível em: <https://ilostat.ilo.org/topics/wages/>. Acesso em: 10 maio. 2020

INSTITUTO NACIONAL DE ESTUDOS E PESQUISAS EDUCACIONAIS ANÍSIO TEIXEIRA. Sinopses Estatísticas da Educação Superior 2018. Brasília: INEP, 2019. Disponível em: <http://portal.inep.gov.br/web/ guest/sinopses-estatisticas-da-educacao-superior>. Acesso em: 6 nov. 2019.

ISAZA VALENCIA, L. Estilos de Aprendizaje: una apuesta por el desempeño académico de los estudiantes en la Educación Superior. Revista Encuentros, v. 12, n. 2, 14 nov. 2014. 
KUH, G. D.; KINZIE, J.; BUCKLEY, J. A. What Matters to Student Success: A Review of the Literature: Commissioned Report for the National Symposium on Postsecondary Student Success: Spearheading a Dialog on Student Success. [s.l.] Nationa postsecondary education coopetarite, 2006.

MARTINS, I.; MARTINS, I. Educação em Ciências e Educação em Saúde: breves apontamentos sobre histórias, práticas e possibilidades de articulação. Ciência \&amp; Educação (Bauru), v. 25, n. 2, p. 269-275, abr. 2019.

NORO, L. R. A. et al. Condições sociais, escolarização e hábitos de estudo no desempenho acadêmico de concluintes da área da saúde. Trabalho, Educação e Saúde, v. 17, n. 2, 2019.

OSTI, A.; ALMEIDA, L. S. Satisfação académica no Ensino Superior. In: ALMEIDA, L. S. (Ed.). . Estudantes do Ensino Superior: Desafios e Oportunidades. Psicologia \& Educação. Braga: ADIPSIEDUC, 2019. p. 99-114.

OYARZÚN ITURRA, G. et al. Habilidades sociales y rendimiento académico: una mirada desde el género. Acta Colombiana de Psicología, v. 15, n. 2, p. 21-28, dez. 2012.

PIOTTO, D. C.; NOGUEIRA, M. A. Incluindo quem? Um exame de indicadores socioeconômicos do Programa de Inclusão Social da USP. Educação e Pesquisa, v. 42, n. 3, p. 625-649, set. 2016.

RICHARDSON, M.; ABRAHAM, C.; BOND, R. Psychological correlates of university students' academic performance: A systematic review and meta-analysis. Psychological Bulletin, v. 138, n. 2, p. 353-387, 2012.

RODRIGUES JÚNIOR, J. F. Avaliação do estudante universitário. São Paulo: Senac, 2009.

SAVIANI, D. A expansão do ensino superior no brasil: mudanças e continuidades. Poíesis Pedagógica, v. 8, n. 2, p. 4-17, 26 abr. 2011.

SOUSA, Z. A. DE S.; FERREIRA, M. A.; MIRANDA, G. J. Teoria da atribuição de causalidade: percepções docentes e discentes sobre os determinantes do desempenho acadêmico. Advances in Scientific and Applied Accounting, v. 1, n. 2, 4 set. 2019.

VALADAS, S. T.; ARAÚJO, A. M. D. DE C.; ALMEIDA, L. S. Abordagens ao estudo e sucesso académico no ensino superior. Revista e-psi : revista eletrónica de psicologia, educação e saúde, v. Ano 4, n. Vol. 1, p. 47-67, jun. 2014. 